International

\title{
Evaluation of the Incidence of Major Adverse Cardiac and Cerebrovascular Events after Percutaneous Coronary Intervention or Coronary Artery Bypass Graft on Proximal Left Anterior Descending Artery with and without Other Coronary Arteries Involvement
}

\author{
Behshad Naghshtabrizi ${ }^{1, *}$, Zahra Sohrabi $^{1}$, Farzad Emami $^{1}$, Babak Manafi ${ }^{2}$, Shafee Membari ${ }^{1}$
}

${ }^{1}$ Department of Cardiology, Ekbatan University Hospital, Hamadan University of Medical Sciences, Hamadan, IR Iran

${ }^{2}$ Department of Cardiac Surgery, Ekbatan University Hospital, Hamadan University of Medical Sciences, Hamadan, IR Iran

\begin{tabular}{l}
\hline A R T I C L E I N F O \\
\hline Article Type: \\
Research Article \\
\hline Article History: \\
Received: 21 Dec 2014 \\
Revised: 16 Jul 2015 \\
Accepted: 19 Aug 2015 \\
\hline
\end{tabular}

\section{Keywords:}

Percutaneous Coronary Intervention

Coronary Artery Bypass

Coronary Artery Disease

\begin{abstract}
A B S T R A C T
Background: Major Adverse Cardiac and Cerebrovascular Events (MACCE) include cardiac death, nonfatal Myocardial Infarction (MI), cerebrovascular events, and Target Vessel Revascularization (TVR) that may happen after Percutaneous Coronary Intervention (PCI) or Coronary Artery Bypass Graft (CABG).

Objectives: This study aimed to compare the incidence of MACCE between the patients who underwent PCI or CABG due to significant Left Anterior Descending (LAD) stenosis with and without other coronary arteries involvement.

Patients and Methods: This randomized mixed cohort study was conducted on 400 patients with Coronary Artery Disease (CAD), 200 with proximal LAD stenosis and 200 with non-proximal LAD stenosis, selected through census. Half of each group underwent PCI and the rest underwent CABG. The participants were followed at fixed intervals after the procedure and the incidence of MACCE was documented in their checklists. Then, relative risk index was used to compare the two groups regarding the incidence of MACCE. Statistical analysis was done using the Stata software, version 11 and $\mathrm{P}<0.05$ was considered to be statistically significant.

Results: The study participants included 281 males and 119 females with the mean age of $61.36 \pm 10.66$ years. The results showed a significant difference between the two groups regarding the incidence of MACCE $(31.58 \%$ vs. $3 \%, \mathrm{P}=0.001)$. However, no significant difference was found between the effects of PCI with drug eluting stent and CABG on proximal LAD ( $3.70 \%$ vs. $3 \%, \mathrm{P}=1.00)$.

Conclusions: Considering the incidence of MACCE, CABG had no superiority over DES for proximal LAD stenosis.
\end{abstract}

Implication for health policy/practice/research/medical education:

This randomized mixed cohort study was performed to evaluate the rate of MACCE in the patients with proximal or non-proximal LAD lesions with or without other coronary arteries involvement who underwent PCI or CABG. The study results revealed similar rates of MACCE among the patients with proximal LAD stenosis who underwent PCI with DES or CABG.

\section{Background}

Over the past decade, cardiovascular disease has emerged as the single most important cause of death worldwide. In 2010, cardiovascular disease caused an estimated 16 million deaths and led to 293 million disability-adjusted life-years

*Corresponding author: Behshad Naghshtabrizi, 10 Taleghani Street, 65168, Hamadan, Iran. Cellphone: +98-9123493924, Fax: +98-8138268034,

E-mail: behshadnaghshtabrizi@yahoo.com lost (1). Similar to many developed countries, low- and middle-income countries confront this health problem. Patients who suffer from Coronary Artery Disease (CAD) usually have specific symptoms, such as exertional chest discomfort or dyspnea on exertion. According to clinical manifestation and/or noninvasive tests, selective coronary angiography may be needed and based on the results, treatment options include medical therapy, Percutaneous 
Coronary Intervention (PCI), and Coronary Artery Bypass Graft (CABG).

Generally, the available stents for PCI are mainly divided into two groups: Bare Metal Stent (BMS) and Drug Eluting Stent (DES). Currently, BMS is being used in $10-30 \%$ of the patients undergoing PCI. This is most often due to patients' inability to take aspirin and plavix for long time due to any reason, significant stenosis in large coronary arteries $(>4$ $\mathrm{mm}$ ), and the setting of acute Myocardial Infarction (MI) (2). Instent restenosis defined as more than 50\% narrowing in the lumen of the stent site is more common with BMS compared to DES. On the other hand, stent thrombosis is more common with DES (2).

Another option for revascularization is $\mathrm{CABG}$, which was first introduced in 1964. CABG is performed offpump or on-pump during which, Left Internal Mammary Artery (LIMA) or Saphenous Vein Grafts (SVG) are grafted to native stenotic vessels. PCI and CABG have some advantages and disadvantages. PCI is less invasive, is accompanied by shorter length of hospital stay, is easily repeated, and is effective in relieving symptoms. Nevertheless, its main disadvantages include restenosis, high incidence of incomplete revascularization, relative inefficiency in patients with severe left ventricular dysfunction, and less favorable outcomes in patients suffering from diabetes. On the other hand, the main advantages of CABG are improved survival in certain subsets, ability to achieve complete revascularization, and wider applicability (anatomic subsets). However, higher morbidity and higher risk of stroke are the principal disadvantages of CABG. In patients with Single Vessel Disease (SVD), if anatomically suitable, $\mathrm{PCI}$ is preferred over CABG. In patients with 2-Vessel Disease (2VD) also, if the patient accepts the possibility of chest pain recurrence or the need for Target Vessel Revascularization (TVR) in future, PCI should be provided. However, CABG is the preferred choice in 3-Vessel Disease (3VD) or left main lesion, especially with reduced Left Ventricular Ejection Fraction (LVEF) (3).

Significant proximal Left Anterior Descending (LAD) stenosis can be responsible for the ischemia of $40-50 \%$ of the myocardium (4-6). Patients with proximal LAD occlusion in association with low LVEF have been reported to have a 19-fold higher mortality compared to the general population (7). CABG provides a survival benefit when significant proximal LAD stenosis is present and there is a tendency to refer patients for $\mathrm{CABG}$ due to the idea that instent restenosis is more prevalent in this type of lesion.

Despite this information, how to manage this group of patients is still a dilemma. Even though significant nonproximal LAD stenosis may jeopardize the myocardium less, it is of particular importance. Involvement of other coronary arteries in addition to LAD involvement may affect the treatment option. Major Adverse Cardiac and Cerebrovascular Events (MACCE) include cardiac death, nonfatal MI, cerebrovascular events, and need for TVR after CABG or PCI (8).

\section{Objectives}

The present study aims to compare CABG and PCI regarding the incidence of MACCE in patients with proximal or non-proximal LAD lesion with and without other coronary arteries involvement.

\section{Patients and Methods}

This randomized mixed cohort study was performed at Ekbatan Hospital, Hamadan, Iran between April 2010 and April 2013. Patients who were selected for Selective Coronary Angiography (SCA) based on their symptoms or high-risk positive noninvasive tests were admitted from emergency room and hospital clinic.

\subsection{Study Population}

Among the patients who were candidate for SCA, 400 were enrolled into this study based on the main inclusion criterion; i.e., significant proximal or non-proximal LAD stenosis with or without other coronary arteries involvement. In general, luminal narrowing equal to or greater than $70 \%$ in LAD, Left Circumflex (LCX), and Right Coronary Arteries (RCA) is considered to be significant. Therefore, 200 patients with proximal LAD stenosis and 200 with non-proximal LAD stenosis were entered into the study through census. Half of each group underwent $\mathrm{PCI}$ and the rest underwent $\mathrm{CABG}$. The decision for PCI or CABG depended on the cardiologist's opinion that was based on the current guidelines, anatomy of the vessel, patient's condition and comorbidities, and patient's preference in some cases. For each patient, a checklist was completed, which consisted of personal information, risk factors, LVEF, number of involved vessels, type of stent (if PCI was the treatment), and MACCE. Every participant also signed a written informed consent. If PCI was considered for proximal LAD, DES was the choice. PCI was performed using standard interventional techniques. Diameter and length of the lesions were assessed by two-dimensional Quantitative Coronary Angiography (QCA). The size and type of the selected stents for each patient were at the discretion of the interventionalist performing the procedure according to the last European Society of Cardiology (ESC) guideline on DES and BMS usage.

\subsection{Two-Dimensional $Q C A$}

This technique is based on contrast coronary angiogram obtaining parameters that objectively quantify the coronary lumen measuring the significance of a coronary stenosis. To obtain the most comprehensive data and avoid foreshortening or underestimation of stenosis severity, at least two projections orthogonal to each other and both perpendicular were analyzed with automated edge detection system (QAngio XA, Medis, Leiden, Netherlands).

All the patients received $300-600 \mathrm{mg}$ clopidogrel, $325 \mathrm{mg}$ aspirin, and $40 \mathrm{mg}$ atorvastatin orally before the procedure. During the procedure, weight-adjusted unfractionated heparin was given with a bolus dose of $100 \mathrm{unit} / \mathrm{kg}$ to achieve activated clotting time of $250-300$ seconds (HemoTech method). After the procedure, all the patients were treated with $75 \mathrm{mg}$ clopidogrel daily for 6 months in the BMS group and for at least 1 year in the DES group. Also, $40 \mathrm{mg}$ atorvastatin daily combined with 325 $\mathrm{mg}$ aspirin was prescribed for 1 month for the BMS and for 
3-6 months for the DES group. Finally, all the patients had to take $80 \mathrm{mg}$ aspirin daily for their whole lives.

Considering the patients undergoing CABG, LIMA was inserted on LAD in all the cases and, if needed, SVGs were inserted on LCX and RCA. In these patients, median sternotomy was performed first. Then, LIM A was harvested (pedicled graft) from the musculophrenic and superior epigastric arteries up to the upper margin of the first rib or higher. In some cases also, saphenous veins were harvested from one or two legs. After systemic heparinization, aortic cannula and two-stage venous cannula were introduced. Cardiopulmonary bypass was then initiated and cardioplegia was used. The LIMA and saphenous veins were anastomosed by 8 - 0 and $7-0$ prolen. The patients were visited and followed at 6-month and 1-, $2-$, and 3-year intervals depending on the time they had received the treatment. During this period, the patients who had complaints about new onset low threshold exertional, resting angina, or equivalent angina were directly referred for SCA. Noninvasive tests were done when required and if the test results were positive, SCA was performed. The patients who did not attend the routine visits were followed by telephone. If there was a new complaint or an admission in another center, they were requested to be visited at the hospital clinic for better evaluation.

The endpoint of our study was MACCE, including cardiac death, nonfatal MI (due to in- stent thrombosis), cerebrovascular events, and TVR. TVR was defined as either surgical or percutaneous reintervention driven by $\geq 50 \%$ diameter stenosis by quantitative analysis with ischemic symptoms or $\geq 70 \%$ diameter stenosis with or without documented ischemia. In-stent thrombosis was categorized as definite (an acute coronary syndrome with angiographic or autopsy evidence of thrombus or occlusion adjacent to a stent), probable (unexplained death within 30 days after stent implantation or AMI involving the target vessel territory without angiographic confirmation), and possible (any unexplained death beyond 30 days after the procedure) according to the Academic Research Consortium (ARC) criteria. Incidence of MACCE, if happened, was also documented in the patients' checklists.

\subsection{Statistical Analysis}

The study groups were compared regarding the incidence of MACCE using relative risk index. Besides, chi-square and/or Fisher's exact test was employed to compare the proportions. All the analyses were done using Stata software, version 11 and $\mathrm{P}<0.05$ was considered to be statistically significant.

\section{Results}

In this study, the patients were divided into four groups depending on involvement of proximal or non-proximal LAD and type of treatment (PCI or CABG). According to the results, 281 patients $(70 \%)$ were male and 119 ones $(30 \%)$ were female, with the mean age of $61.36 \pm 10.66$ years. The most common risk factor was hypertension (43.5\%). The mean LVEF was $47.35 \pm 7.41 \%$. Besides, the mean time interval of MACCE occurrence after the treatment was $13.83 \pm 64.09$ days in the PCI group and $3.02 \pm 24.5$ days in the CABG group $(\mathrm{P}=0.053)$ (Table 1$)$. During the follow-up, 19 patients experienced an event; 3 non-cardiac events and 16 MACCE. Among these patients, 10 belonged to the PCI group and 6 to the CABG group (P $=0.025)$. MACCE also occurred in 9 patients with proximal LAD lesions ( 5 cardiac deaths and 4 patients needed TVR)

\begin{tabular}{|c|c|c|c|c|c|}
\hline Variables & PCI-P & PCI-NP & CABG-P & CABG-NP & P value \\
\hline Age (year), Mean \pm SD & $59.46 \pm 11.63$ & $61.30 \pm 11.52$ & $63.90 \pm 9.67$ & $60.81 \pm 9.83$ & 0.029 \\
\hline Sex (male), n (\%) & $67(67.00)$ & $65(65.00)$ & $73(73.00)$ & $76(76.00)$ & 0.288 \\
\hline Duration of follow-up (day) & $712.57 \pm 273.49$ & $742.20 \pm 278.36$ & $858.94 \pm 253.87$ & $594.30 \pm 281.45$ & 0.001 \\
\hline Ejection fraction (\%) & $48.87 \pm 7.45$ & $46.85 \pm 7.38$ & $46.25 \pm 7.83$ & $47.45 \pm 6.98$ & 0.078 \\
\hline Time of event after procedure (day) & $19.87 \pm 75.33$ & $7.80 \pm 52.85$ & $3.34 \pm 32.99$ & $2.70 \pm 16.01$ & 0.053 \\
\hline History of diabetes & $26(26.00)$ & $19(19.00)$ & $26(26.00)$ & $24(24.00)$ & 0.613 \\
\hline History of smoking & $30(30.00)$ & $47(47.00)$ & $36(36.00)$ & $28(28.00)$ & 0.022 \\
\hline History of hypertension & $38(38.00)$ & $43(43.00)$ & $48(48.00)$ & $45(45.00)$ & 0.341 \\
\hline History of hyperlipidemia & $22(22.00)$ & $22(22.00)$ & $21(22.00)$ & $23(23.00)$ & 0.990 \\
\hline History of PVD & $2(2.00)$ & $2(2.00)$ & $2(2.00)$ & $6(6.00)$ & 0.248 \\
\hline History of MI & $12(12.00)$ & $16(16.00)$ & $22(22.00)$ & $21(21.00)$ & 0.218 \\
\hline History of stroke & $3(3.00)$ & $7(7.00)$ & $8(8.00)$ & $9(9.00)$ & 0.348 \\
\hline Incomplete revascularization & $18(18.00)$ & $12(12.00)$ & $10(10.00)$ & $10(10.00)$ & 0.269 \\
\hline Stent thrombosis (sub-acute) & $2(2.00)$ & $0(0.00)$ & $0(0.00)$ & $0(0.00)$ & 0.110 \\
\hline Number of involved vessels & & & & & 0.001 \\
\hline I & $80(80.00)$ & $81(81.00)$ & $14(14.00)$ & $2(2.00)$ & \\
\hline II & $18(18.00)$ & $19(19.00)$ & $30(30.00)$ & $26(26.00)$ & \\
\hline III & $2(2.00)$ & $0(0.00)$ & $56(56.00)$ & $72(72.00)$ & \\
\hline Setting & & & & & 0.121 \\
\hline Chronic stable angina & $16(16.00)$ & $18(18.00)$ & $10(10.00)$ & $79(79.00)$ & \\
\hline Acute coronary syndrome & $78(78.00)$ & $65(65.00)$ & $82(82.00)$ & $13(13.00)$ & \\
\hline ST-elevation MI elective & $6(5.00)$ & $17(16.00)$ & $8(8.00)$ & $8(8.00)$ & \\
\hline
\end{tabular}

Abbreviations: PCI-P, percutaneous coronary intervention-proximal LAD; PCI-NP, percutaneous coronary intervention-non proximal LAD; CABG-P, coronary artery bypass graft proximal LAD; CABG-NP, coronary artery bypass graft non-proximal LAD; PVD, peripheral vascular disease 
and one patient with non-proximal LAD lesion (one cardiac death) who underwent PCI. MACCE, too, occurred in 3 patients with proximal LAD lesions ( 3 cardiac deaths) and 3 patients with non-proximal LAD lesions (one cardiac death and 2 patients needed TVR) who underwent CABG. The results only revealed a significant difference between the groups with proximal LAD and non-proximal LAD that underwent PCI $(\mathrm{P}=0.018)$.

Among the patients with proximal LAD stenosis in the PCI group, 81 and 19 received DES and BMS, respectively. BMS was used for the patients who could not afford DES and had refused $\mathrm{CABG}$. In the PCI group with proximal LAD lesions, 9 cases of MACCE were reported; 3 from the 81 patients $(3.70 \%)$ with DES and 6 from the 19 patients (31.58\%) with BMS ( $\mathrm{P}=0.001)$ (Table 2). The results showed no significant difference between the patients with proximal LAD lesions who underwent PCI with DES and CABG regarding the incidence of MACCE $(\mathrm{P}=1.000)$. However, a significant difference was observed between the patients with proximal LAD lesion who underwent PCI with BMS and CABG in this regard $(\mathrm{P}=0.001)$. In the PCI group, proximal LAD lesion with other vessels involvement occurred in 20 patients among whom, 5 experienced MACCE. From these cases, one had DES and 4 had BMS ( $\mathrm{P}=0.041)$. Out of the four study groups, the results only showed a significant difference between the patients with SVD and Multi-Vessel Disease (MVD) in the group with proximal LAD lesions who underwent PCI regarding the incidence of MACCE $(\mathrm{P}=0.015)$. However, no significant difference was observed among the other three groups in this regard (Table 3).

\section{Discussion}

Regarding the progressive increase in the prevalence of cardiovascular risk factors and cardiovascular diseases, studies leading to better solutions for CAD treatment are of significant importance. Evidence has indicated that DES is superior to BMS due to lower rates of in-stent restenosis. However, it has some disadvantages, including higher rates of stent thrombosis, higher price, and need for dual antiplatelet therapy for a longer duration compared to BMS. The present study aimed to compare the patients with proximal LAD stenosis with and without other vessels involvement regarding the incidence of MACCE after PCI and CABG. In this study, the median age of the patients was not too high and the median percent of LVEF was not too low to affect the results. However, subgroup analysis was not done, which can be considered to be a limitation to the study.

Rodriguez et al. compared PCI and CABG in MVD patients with proximal LAD involvement. In that study, patients with MVD and significant stenosis of the proximal LAD were randomly assigned to PCI or CABG. The two groups had similar survival and survival with freedom from MI at long-term follow-up. However, repeated revascularization procedures were higher in the PCI group. On the other hand, no significant early and late differences were observed between the two groups concerning total MACCE. In that study, target vessels were stented with Gianturco Roubin II stent (Cook Inc, Bloomington, Indiana, USA) as the primary device. CABG procedure was also performed by standard surgical techniques. Complete revascularization was achieved, when possible, by using arterial conduits and reverse saphenous vein grafts (9).

In the current study, PCI was performed with a newer generation of BMS and DES (strong point) and CABG was done with the same standard surgical technique. Follow-up period and the results of both studies were also the same.

Drenth et al. evaluated the outcome of stent and offpump LIMA on proximal LAD. According to the findings,

\begin{tabular}{llll}
\hline \multicolumn{2}{l}{ Table 2. Comparison of PCI with DES and BMS Regarding the Rate of MACCE } \\
\hline Stent on Proximal LAD & \multicolumn{2}{c}{ MACCE } & Total \\
\cline { 2 - 4 } & MACCE+ & MACCE- & 81 \\
DES,n(\%) & $3(3.70)$ & $78(96.30)$ & 19 \\
BMS, $\mathbf{n}(\%)$ & $6(31.58)$ & $13(68.42)$ & 100 \\
Total,n(\%) & $9(9.00)$ & $91(91.00)$ & 0.001 \\
\hline
\end{tabular}

Fisher's exact test was employed to compare the proportions.

\begin{tabular}{|c|c|c|c|c|}
\hline Number of Involved Vessels & MACCE+ & MACCE- & Total & P value \\
\hline \multicolumn{5}{|l|}{ PCI proximal } \\
\hline SVD, n (\%) & $4(5.00)$ & $76(95.00)$ & 80 & 0.015 \\
\hline MVD, n (\%) & $5(25.00)$ & $15(75.00)$ & 20 & \\
\hline \multicolumn{5}{|l|}{ PCI non-proximal } \\
\hline SVD, n (\%) & $1(1.22)$ & $81(98.78)$ & 82 & 1.00 \\
\hline MVD, n (\%) & $0(0.00)$ & $18(100.00)$ & 18 & \\
\hline \multicolumn{5}{|l|}{ CABG Proximal } \\
\hline SVD, n (\%) & $0(0.00)$ & $13(100.00)$ & 13 & 1.00 \\
\hline MVD, n (\%) & $3(3.45)$ & $84(96.55)$ & 87 & \\
\hline \multicolumn{5}{|l|}{ CABG non- Proximal } \\
\hline SVD, n (\%) & $0(0.00)$ & $2(100.00)$ & 2 & 1.00 \\
\hline MVD, n (\%) & $3(3.06)$ & 95(96.94) & 98 & \\
\hline
\end{tabular}

Fisher's exact test was employed to compare the proportions. 
patients with an isolated high-grade lesion of the proximal LAD had a significantly better 4-year clinical outcome after off-pump coronary bypass grafting than after PCI. In that study, only proximal LAD lesion was taken into account, CABG was done off-pump, PCI was done with BMS, and dual antiplatelet therapy included aspirin and ticlopidine (4). These might justify the discrepancy between the results of their study and ours.

Moshkovitz et al. also compared the outcome of CABG and DES on proximal LAD. The results showed no significant difference regarding the 30-day mortality rate, but recurrence of angina and TVR was higher in the DES group. In that study, Cypher (first DES generation) was used in the PCI procedure and follow-up period was only one year4. These were the main differences between that study and the present one, which could explain the difference between the results.

Furthermore, Blazek et al. assessed the 10-year safety and effectiveness of PCI and Minimally Invasive Direct Coronary Artery Bypass (MIDCAB) surgery in treatment of proximal LAD lesions. At the 10-year follow-up, PCI and MIDCAB yielded similar long-term outcomes regarding the primary composite clinical endpoint. However, TVR was more frequent in the PCI group. In that research, only BMS was used in the PCI group, but MIDCAB was preferred in the $\mathrm{CABG}$ group due to isolated significant proximal LAD stenosis (10). These were the main differences between that study and the current one.

In our study, the patients who underwent PCI with BMS experienced MACCE more in comparison to those who underwent $\mathrm{CABG}$. This was in agreement with the results of the study conducted by $\mathrm{Wu} \mathrm{C}$ (11). Experts also believe that PCI on proximal LAD should be performed with DES. Similarly, Jones et al. reported lower MACCE rates with no increase in the incidence of stent thrombosis while using DES in comparison to BMS in significant proximal LAD stenosis (12).

Another study that was performed by Blazek et al. also revealed similar long-term outcomes regarding composite end point of death, MI, and TVR in the patients who underwent PCI with Sirolimus Eluting Stent (SES) and MIDCAB on isolated proximal LAD lesions. Nonetheless, the rate of TVR was higher in the PCI group (13).

A meta-analysis of 12 studies including more than 2000 patients revealed comparable survival rates after MIDCAB and PCI with DES on proximal LAD stenosis, with significantly higher need for revascularization in the PCI group due to angina recurrence (14).

Garg et al. also indicated that at 5-year follow-up, CABG had comparable mortality, death, stroke, and MI rates and superior efficacy in terms of reducing repeated revascularization compared to BMS and SES in treatment of patients with MVD involving the proximal LAD (15).

Moreover, Nejat et al. demonstrated that changes in Total Cholesterol (TC), Triglyceride (TG), non-High Density Lipoprotein Cholesterol (non HDL-C), TC/HDL-C, and TG/HDL-C were independent predictors of Coronary Heart Disease (CHD) events (16). In addition, maintained dyslipidemia was a strong predictor of CHD events. Thus, controlling this major risk factor could certainly affect further prevention of cardiac events after PCI or CABG.

The findings of the present study showed no significant difference between PCI with DES and CABG on proximal LAD stenosis regarding the incidence of MACCE $(\mathrm{P}=$ 1.000). However, a significant difference was found between CABG and PCI with BMS in this respect $(\mathrm{P}=0.001)$. The results also indicated the superiority of DES to BMS on proximal LAD stenosis. This implies that if the anatomy and patient's conditions are suitable for PCI on proximal LAD stenosis, DES should be the choice and CABG be saved for later stages. Additionally, when PCI was performed on proximal LAD, the incidence of MACCE was significantly higher in MVD compared to SVD. However, this difference was not detected in the other 3 groups.

Our study findings demonstrated that the mean number of days before occurrence of MACCE was significantly shorter in the CABG group compared to the PCI group (3.02 and 13.38 days, respectively) $(\mathrm{P}=0.053)$. This means that the first few days after CABG are more critical and later MACCE is more prevalent in PCI patients.

Different types of DES and mean duration of followup in the aforementioned studies led to various results in comparison to the current study. Thus, using the new generation of DES on significant proximal LAD lesions might be responsible for the negligible incidence of MACCE and its components, including TVR, in our study. Considering the incidence of MACCE, CABG had no superiority to PCI with DES on proximal LAD stenosis. In addition, DES was related to lower rates of MACCE compared to BMS.

\section{Acknowledgements}

The authors are grateful for the staff in the Departments of interventional cardiology and cardiac surgery, Ekbatan University Hospital for their assistance.

\section{Authors' Contribution}

Z Sohrabi and S Membary collected and analyzed the data, B Naghshtabrizi and F Emami managed and prepared the manuscript, B Naghshtabrizi reviewed and approved the manuscript

\section{Financial disclosure}

There is no financial disclosure.

\section{Funding/Support}

There is no funding/support.

\section{References}

1. The Global Burden of Disease. 2004 update,2008 Available from: www.who.int/evidence/bod.

2. Mann DL, Zipes DP, Libby P, Bonow RO. Braunwald's heart disease: a textbook of cardiovascular medicine. Elsevier Health Sciences; 2014.

3. Morrow D. Stable ischemic heart disease. In: Libby P, Bonow R, Mann D, Zipes D, editors. Braunwald's heart disease: a textbook of cardiovascular medicine. 9th ed. Philadelphia: Saunders; 2012. p. 1239-48.

4. Drenth DJ, Veeger NJ, Grandjean JG, Mariani MA, van Boven AJ, Boonstra PW. Isolated high-grade lesion of the proximal LAD: a stent or off-pump LIMA? Eur J Cardiothorac Surg. 2004;25(4):567-71.

5. Hannan EL, Racz MJ, Walford G, Jones RH, Ryan TJ, Bennett E, et al. Long-term outcomes of coronary-artery bypass grafting versus 
stent implantation. N Engl J Med. 2005;352(21):2174-83.

6. Moshkovitz Y, Mohr R, Braunstein R, Zivi E, Uretzky G, Ben-Gal $\mathrm{Y}$, et al. Revascularization of left anterior descending coronary artery in patients with single and multivessel disease: comparison between off-pump internal thoracic artery and drug-eluting stent. Chest. 2005;128(2):804-9.

7. Valencia J, Berenguer A, Mainar V, Bordes P, Gomez S, Tello A, et $a l$. Two-year follow-up of sirolimus-eluting stents for the treatment of proximal left anterior descending coronary artery stenosis. $J$ Interv Cardiol. 2006;19(2):126-34.

8. King S, Yeong A. Interventional cardiology. New York: McGrow Hill; 2007.

9. Rodriguez A, Rodriguez Alemparte M, Baldi J, Navia J, Delacasa A, Vogel D, et al. Coronary stenting versus coronary bypass surgery in patients with multiple vessel disease and significant proximal LAD stenosis: results from the ERACI II study. Heart. 2003;89(2):184-8.

10. Blazek S, Holzhey D, Jungert C, Borger MA, Fuernau G, Desch S, et al. Comparison of bare-metal stenting with minimally invasive bypass surgery for stenosis of the left anterior descending coronary artery: 10-year follow-up of a randomized trial. JACC Cardiovasc Interv. 2013;6(1):20-6.

11. Wu C, Zhao S, Wechsler AS, Lahey S, Walford G, Culliford AT, et al. Long-term mortality of coronary artery bypass grafting and bare-metal stenting. Ann Thorac Surg. 2011;92(6):2132-8.
12. Jones DA, Rathod KS, Gallagher S, Weerackody R, Knight CJ, Rothman MT, et al. Deployment of drug-eluting stents for isolated proximal lad disease is associated with lower major adverse cardiac events and no increase in stent thrombosis when compared with bare metal stents: a 5-year observational cohort study. Catheter Cardiovasc Interv. 2013;81(6):E237-44.

13. Blazek S, Rossbach C, Borger MA, Fuernau G, Desch S, Eitel I, et al. Comparison of sirolimus-eluting stenting with minimally invasive bypass surgery for stenosis of the left anterior descending coronary artery: 7-year follow-up of a randomized trial. JACC Cardiovasc Interv. 2015;8(1 Pt A):30-8.

14. Deo SV, Sharma V, Shah IK, Erwin PJ, Joyce LD, Park SJ. Minimally invasive direct coronary artery bypass graft surgery or percutaneous coronary intervention for proximal left anterior descending artery stenosis: a meta-analysis. Ann Thorac Surg. 2014;97(6):2056-65.

15. Garg S, Sarno G, Gutierrez-Chico JL, Garcia-Garcia HM, GomezLara J, Serruys PW. Five-year outcomes of percutaneous coronary intervention compared to bypass surgery in patients with multivessel disease involving the proximal left anterior descending artery: an ARTS-II sub-study. EuroIntervention. 2011;6(9):1060-7.

16. Nejat A, Mirbolouk M, Mohebi R, Hasheminia M, Tohidi M, Saadat $\mathrm{N}$, et al. Changes in lipid measures and incident coronary heart disease: Tehran Lipid \& Glucose Study. Clin Biochem. 2014;47(13-14):1239-44. 